\title{
Self-Quarantine Noncompliance During the COVID-19 Pandemic in South Korea
}

\author{
Sukhyun Ryu, MD, PhD; Youngsik Hwang, PhD; Hongbi Yoon; Byung Chul Chun, MD, PhD
}

ABSTRACT

Objectives: In South Korea, many individuals were self-quarantined for the coronavirus disease 2019 (COVID-19) after the quarantine criteria were extended to all overseas travelers. This study was conducted to identify the noncompliance rate of self-quarantine for COVID-19 cases and assess the impact of a 1-strike out policy and an increased amount of penalty for the violating self-quarantine in South Korea.

Methods: The self-quarantine noncompliance rate for COVID-19 was examined using publicly available data. We collected the daily number of quarantine and quarantine violation cases from March 22 to June 10, 2020. A Poisson regression analysis was conducted to identify the impact of additional sanctions for the quarantine violation.

Results: The median number of individuals quarantined per day was 36,561 (interquartile range, 34,40841,961). The median number of daily self-quarantine violations was 6 (range, $0-13$ ). The median rate of self-quarantine violations was 1.6 per 10,000 self-quarantined individuals (range, 0.0-8.0 per 10,000 self-quarantined individuals). The additional sanction has no significant impact on the number of violations among quarantine individuals $(P=0.99)$.

Conclusions: The additional sanction for the violation of quarantined individuals did not reduce the selfquarantine violations. Further studies are warranted to strengthen the compliance of self-quarantine for future pandemics.

Key words: compliance, coronavirus, COVID-19, Korea, quarantine

$\mathrm{T}$ he severe acute respiratory syndrome coronavirus 2 (SARS-CoV-2) has affected South Koreans since January 20, 2020. ${ }^{1}$ To prevent the spread of SARS-CoV-2, the Korean government has implemented an unprecedented, large-scale home-quarantine for suspected or confirmed cases of coronavirus disease 2019 (COVID-19). ${ }^{2}$ In the early epidemics of COVID-19, financial aids to the those quarantined cases and compensation for the employer for the wage loss were provided under the Korean Infectious Disease Control and Prevention Act (Table 1). ${ }^{3}$ In South Korea, to prevent seeding new SARS-CoV-2 infection, any individual who has traveled from a country with a high COVID-19 infection risk is also obliged to self-quarantine. ${ }^{4}$ The quarantined individuals are asked to comply with self-quarantine and are monitored for 14 days. Since April 1, 2020, all overseas travelers have been included in the selfquarantine program, which has greatly increased the number of quarantined individuals in South Korea (Figure 1A). The self-quarantine individuals have been actively monitored by a mobile application or phone-call twice a day and the public health workers sometimes randomly visit the quarantined place in person (Table 1). ${ }^{5}$ On April 5, 2020, the Korean government implemented a "1-strike out policy" and increased the penalty from a maximum of 3 million to 10 million Korean Won (8273 USD) in fines to further discourage quarantine violation. A previous study demonstrated that self-quarantine compliance for the case of COVID-19 is crucial for preventing local transmission $^{6}$; however, there is no literature measuring the exact noncompliance rate of self-quarantine for COVID-19 cases.

\section{METHODS}

This study did not require the institutional review board approval or informed consent, because the data had already been published by the Korean Ministry of Interior and Safety. ${ }^{7}$ To obtain the noncompliance rate of self-quarantine for COVID-19 cases in South Korea, we collected the daily number of quarantine and quarantine violation cases from March 22 to June 10, 2020. To assess the impact of a 1-strike out policy and increased the penalty, the Poisson regression model incorporated with the additional sanctions were used to estimate the intervention effect with the confidence 
Overview of the Korean strategy for encouraging self-quarantine for COVID-19 cases

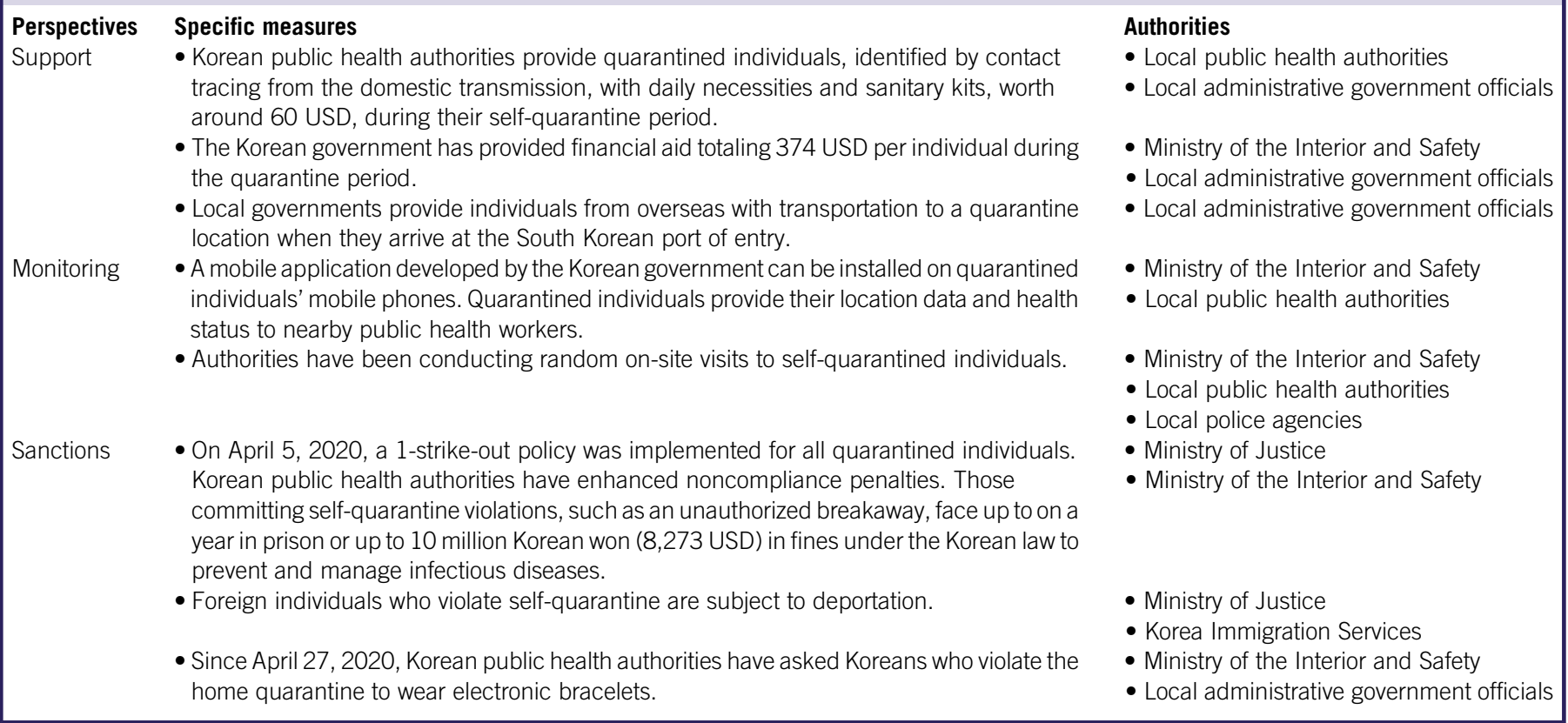

intervals on the daily rate (per 10,000 quarantined cases) and the daily number of quarantine violation. The established Poisson regression model is

$$
\log \left(\mu_{t}\right)=\beta_{0}+\beta_{1} x_{t}
$$

Where, $\mu_{\mathrm{t}}$ represents the count of quarantine violation at day $t$, $\beta_{0}$ and $\beta_{1}$ denote estimate for base level of the quarantine violation and multiplicative effect on the quarantine violation, and $x_{t}$ represents the additional sanctions $\left(x_{t}=0\right.$ from March 22 to April 5, 2020; $x_{t}=1$ from April 6 to June 10, 2020). All statistical analyses were performed in $\mathrm{R}$ version 3.0.2 ( $\mathrm{R}$ Foundation for Statistical Computing, Vienna, Austria).

\section{RESULTS}

The median number of individuals quarantined per day was 36,561 (interquartile range [IQR], 34,408-41,961; range, $8335-59,918)$. The median number of quarantined individuals, including those in contact with suspected and confirmed cases and those who have traveled overseas, was 5127 (IQR, 2451-7568; range, 846-11,053) and 32,041 (IQR, 28,92337,819; range, 0-55,590), respectively (Figure 1A). The median number of daily self-quarantine violations was 6 , with a range of 0-13 (Figure 1B). The median rate of self-quarantine violations was 1.6 per 10,000 quarantined individuals (range, 0.0-8.0 per 10,000) (Figure 1C). We identified a 1-strike out sanction and increased the amount of penalty had not a significant change on the daily violation rate $(P=0.99)$ (Table 2$)$. Furthermore, it had not any significant impact on the number of violations among Koreans $(P=0.91)$ and foreigners $(P=0.81)$.

\begin{tabular}{|c|c|c|c|}
\hline \multicolumn{4}{|c|}{$\begin{array}{l}\text { Results of a Poisson regression analysis by implemen- } \\
\text { tation of a } 1 \text {-strike out policy and increased the amount } \\
\text { of penalty }\end{array}$} \\
\hline arameter & Estimates & $\begin{array}{l}\text { 95\% Confidence } \\
\text { Interval }\end{array}$ & p-value \\
\hline $\begin{array}{l}\text { Rate of quarantine } \\
\text { violationa }\end{array}$ & 0.001 & $-0.23-0.25$ & 0.99 \\
\hline $\begin{array}{l}\text { Number of quarantine } \\
\text { violation of Koreans }\end{array}$ & 0.02 & $-0.24-0.29$ & 0.91 \\
\hline $\begin{array}{l}\text { Number of quarantine } \\
\text { violation of foreigners }\end{array}$ & -0.07 & $-0.59-0.53$ & 0.81 \\
\hline
\end{tabular}

${ }^{\mathrm{a}}$ rate: per 10,000 quarantined individuals

\section{DISCUSSION}

To limit the local transmission of SARS-CoV-2, public compliance with public health measures, such as self-quarantine for suspected or confirmed cases of COVID-19 is essential. ${ }^{6,8} \mathrm{~A}$ previous study demonstrated that a major obstacle to selfquarantine compliance was concern over the loss of income. ${ }^{9}$ To overcome this obstacle, the Korean government developed 3 specific plans early in the epidemic to reimburse, monitor, and sanction the individuals in self-quarantine for COVID-19 (Table 1).

The Korean government's efforts to control the local transmission of SARS-CoV-2 has been positively evaluated among global public health experts; however, the implemented policies, particularly the forced control of people, is necessary to discuss. 


\section{FIGIURE}

The Number of Individuals Quarantining and Individuals Who Have Violated Quarantine in South Korea Between March 22 and June 10,2020 . The vertical dashed line in red indicates the implementation of a 1-strike out policy and an increased penalty for the violation of individuals from self-quarantine. (A) The daily number of quarantined individuals as determined by contact tracing of locals and international travelers. (B) The daily number of violators. (C) The daily noncompliance rate of those in self-quarantine.
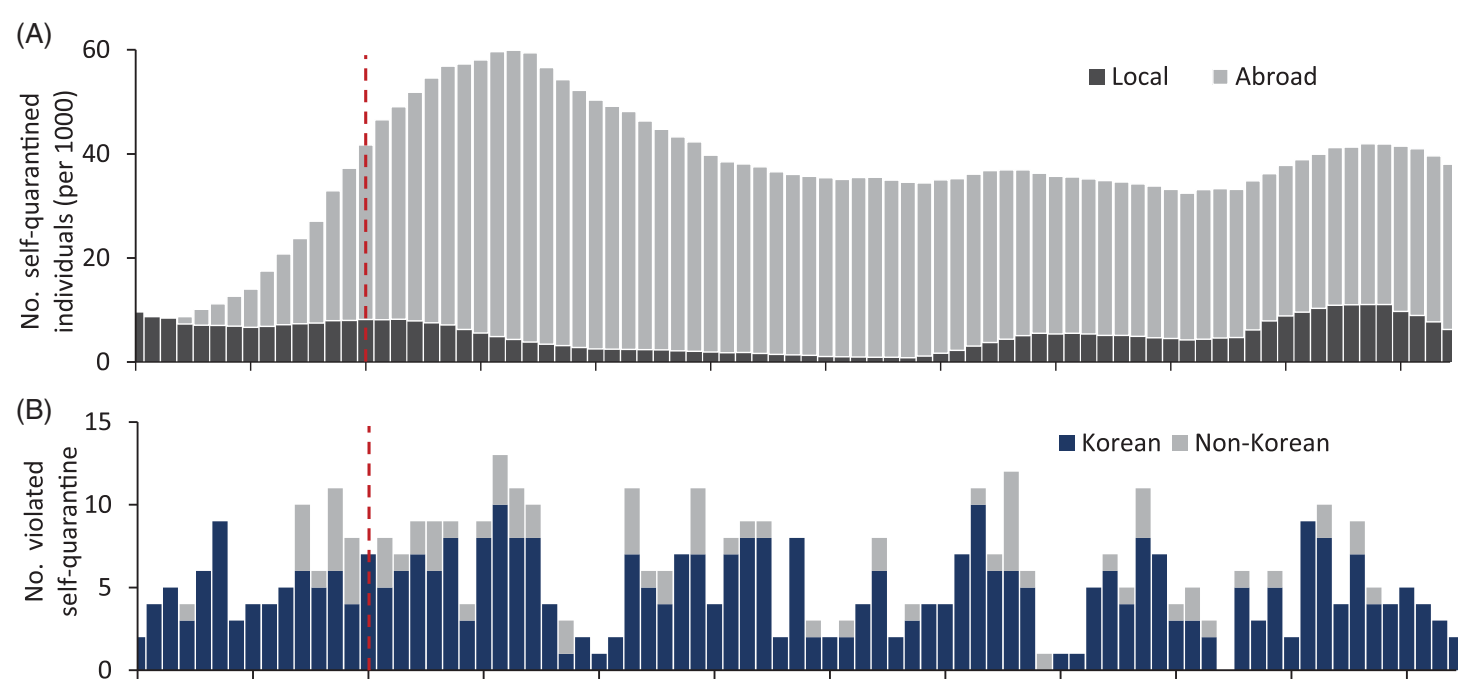

(C)

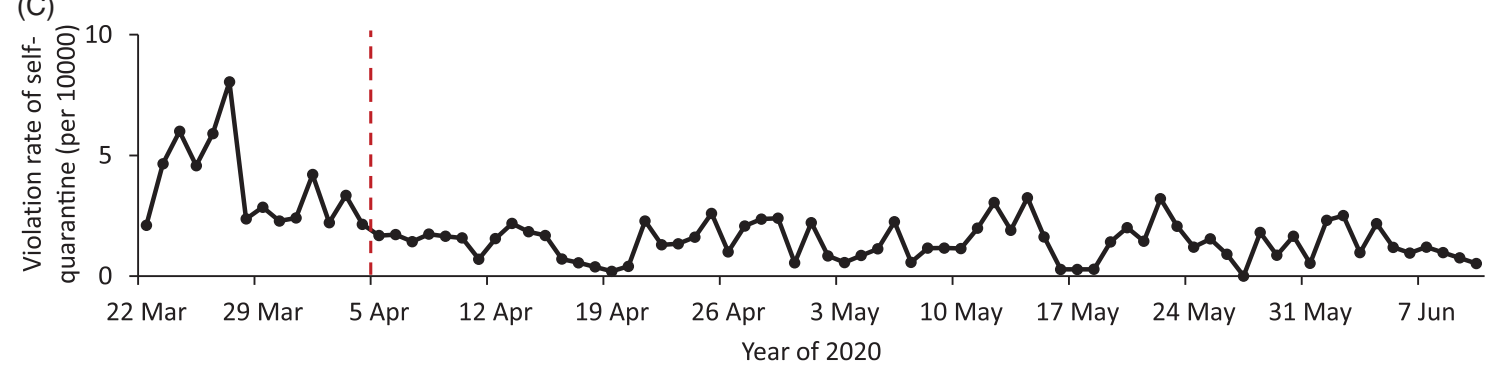

Our findings indicate that additional self-quarantine regulations did not significantly affect the violation rate of quarantine. This signifies the importance of a proper level of policy execution to control the number of violations and helps to decrease unnecessary costs and avoid time-consuming decision-making processes. Our findings also provide a proxy of the self-quarantine noncompliance rate under governmental regulation. However, this study is limited by a lack of demographic data and details on the reasons for violations, which make it challenging to identify the risk factors associated with breaches of self-quarantine rules. Furthermore, additional timeseries data before our study period are required to improve the model fit and accuracy.

As of September 7, 2020, the two COVID-19 epidemic waves identified, ${ }^{10}$ and the violations of self-quarantine are still being reported in South Korea. Further studies including the effective process for delivering comprehensive information about the self-quarantine to the public and appropriate level of policy for discouraging self-quarantine violations are warranted.

\section{CONCLUSIONS}

Our results identified the additional sanctions, including a 1-strike out policy and increased the amount of penalty, had no significant impact on reducing the violation rate of selfquarantine in South Korea. Additional studies are warranted to strengthen quarantine compliance for future epidemics.

\section{About the Authors}

Department of Preventive Medicine, Konyang University College of Medicine, Daejeon, Republic of Korea (Drs Ryu, Hwang, Yoon) and Department of Preventive Medicine, Korea University College of Medicine, Seoul, Republic of Korea (Dr Chun).

Correspondence and reprint requests to Sukhyun Ryu, Department of Preventive Medicine, Konyang University College of Medicine, R707, Myungok-Euihak 
Gwan, 158, Gwanjeodong-ro, Seogu, Daejeon, Republic of Korea, 35365 (e-mail: gentryu@onehealth.or.kr).

\section{Acknowledgments}

The authors thank Dr Sheikh Taslim Ali (The University of Hong Kong) and anonymous reviewers for their constructive comments.

\section{Funding}

This study was financially supported by the Basic Science Research Program through the National Research Foundation of Korea funded by the Korean Ministry of Education (NRF-2020R1I1A3066471). The funder had no role in the design and conduct of the study; collection, management, analysis, and interpretation of the data; preparation, review, or approval of the manuscript; and decision to submit the manuscript for publication.

\section{Data availability Statement}

The study data are available from the corresponding author upon reasonable request.

\section{Ethics Committee Approval}

Because this study was based on publicly available data, the institutional review board approval or informed consent was not required.

\section{Conflict of Interest}

The authors have no conflicts of interest to declare.

\section{REFERENCES}

1. Ryu S, Chun BC, Korean Society of Epidemiology -nCo VTFT. An interim review of the epidemiological characteristics of 2019 novel coronavirus. Epidemiol Health. 2020;42:e2020006.

2. Ryu S, Ali ST, Jang C, et al. Effect of nonpharmaceutical interventions on transmission of severe acute respiratory syndrome coronavirus 2, South Korea, 2020. Emerg Infect Dis. 2020;26(10):2406-2410.

3. Namgoong J. The Republic of Korea's Policy Response to the Covid-19 epidemic in the field of employment and labour relations. http://www. cielolaboral.com/wp-content/uploads/2020/04/republica_de_corea_noticias_ cielo_coronavirus.pdf. Published 2020. Accessed September 9, 2020.

4. Lee H, Kim K, Choi K, et al. Incubation period of 2019 novel the coronavirus disease 2019 (COVID-19) infections in Busan, South Korea. J Infect Chemother. 2020. doi: https://doi.org/10.1016/j.jiac.2020.06.018

5. Kim MS. South Korea is Watching Quarantined Citizens with a Smartphone App. Cambridge, MA: MIT Technology Review; March 6, 2020.

6. Kim HJ, Hwang HS, Choi YH, et al. The delay in confirming COVID-19 cases linked to a religious group in Korea. J Prev Med Public Health. 2020;53(3):164-167.

7. Korean Ministry of the Interior and Safety. Self-quarantined individual management to prevent COVID-19. https://www.mois.go.kr/frt/bbs/type010/ commonSelectBoardArticle.do?bbsId=BBSMSTR_000000000008\&nttId= 77840. Accessed September 9, 2020

8. Ryu S, Ali ST, Lim JS, et al. Estimation of the excess COVID-19 cases in Seoul, South Korea by the students arriving from China. Int J Environ Res Public Health. 2020;17(9):3113.

9. Bodas M, Peleg K. Self-isolation compliance in the COVID-19 era influenced by compensation: findings from a recent survey in Israel. Health Aff (Millwood). 2020;39(6):936-941.

10. Ryu S, Noh E, Ali ST, et al. Epidemiology and control of two epidemic waves of SARS-CoV-2 in South Korea. SSRN. 2020. https://papers.ssrn.com/sol3/ papers.cfm?abstract_id=3687061. Accessed October 26, 2020. 